Habib ur Rehman, Dhananjay Gopal, and Poom Kumam*

\title{
Generalizations of Darbo's fixed point theorem for new condensing operators with application to a functional integral equation
}

https://doi.org/10.1515/dema-2019-0012

Received September 6, 2018; accepted January 27, 2019

\begin{abstract}
In this paper, we provide some generalizations of the Darbo's fixed point theorem associated with the measure of noncompactness and present some results on the existence of the coupled fixed point theorems for a special class of operators in a Banach space. To acquire this result, we define $\alpha-\psi$ and $\beta$ - $\psi$ condensing operators and using them we propose new fixed point results. Our results generalize and extend some comparable results from the literature. Additionally, as an application, we apply the obtained fixed point theorems to study the nonlinear functional integral equations.
\end{abstract}

Keywords: Darbo fixed point theorem, measure of noncompactness, fixed point, coupled fixed point, functional integral equation

MSC: 47H08, 47H09, 47H10

\section{Introduction and preliminaries}

The study of nonlinear integral equations, nowadays, is a subject of interest for many researchers in nonlinear functional analysis. Integral equations arise in many practical problems including potential theory and other physics-related problems. On the other hand, fixed point theory is one of the most effective and fruitful tool used in nonlinear analysis to solve functional integral equations. It's concerned with the conditions for the existence of one or more fixed points of a mapping $T$ from a topological space $X$ into itself. Brouwer [1] established a fixed point result what has become the well-known Brouwer's fixed point theorem for finite dimensional spaces. While in 1922, Banach [2] introduced his celebrated Banach contraction principle for complete metric spaces which guarantee the existence and uniqueness of fixed point. Afterwards, in 1930, Schauder [3] extended the Brouwer's fixed point theorem to infinite dimensional spaces using the condition of compactness. There are many developments in fixed point theory in various directions, one among them is single-valued mappings (see [4-10] and references therein). Furthermore, Kuratowski [11] in 1930, opened up a new direction of research with the introduction of the concept of a measure of noncompactness, which gives the degree of noncompactness for bounded sets. The measure of noncompactness can also be used in the study of single-valued and multivalued mappings, especially in metric and topological fixed point theory. The measure of noncompactness combining with some algebraic arguments is beneficial for studying mathematical formulations, especially solving the existence of solutions of some nonlinear problems under certain situations.

\footnotetext{
Habib ur Rehman: Department of Mathematics, Faculty of Science, King Mongkut's University of Technology Thonburi (KMUTT), 126 Pracha Uthit Rd., Bang Mod,Thung Khru, Bangkok 10140, Thailand; E-mail: hrehman.hed@gmail.com Dhananjay Gopal: National Institute of Technology, Surat- 395007 Gujarat, India; E-mail: gopal.dhananjay@rediffmail.com ${ }^{\star}$ Corresponding Author: Poom Kumam: Department of Mathematics, Faculty of Science, King Mongkut's University of Technology Thonburi (KMUTT), 126 Pracha Uthit Rd., Bang Mod,Thung Khru, Bangkok 10140, Thailand; E-mail: poom.kum@kmutt.ac.th
} 
The Kuratowski and Hausdorff measure of noncompactness [12,13] in a metric space are well-known in the literature and the proof of the Darbo's fixed point result depends upon the technique of the measure of noncompactness. The Darbo's fixed point theorem is a very useful generalization of Schauder's fixed point theorem for noncompact operators which is very helpful to solve differential and integral equations. Due to this fact, researchers are always interested to find the extensions and generalizations of the Darbo's fixed point theorem. Up to now, several papers have been published on the generalization of Darbo's fixed point theorem (see [14-18] and references therein) and on the existence and behaviour of solutions of nonlinear differential and integral equations [19-27] using the technique of measure of noncompactnes.

On the other hand, in metric fixed point theory Samet [9] introduced a nice generalization of Banach fixed point theorem using the Definition 1.1, of $\alpha$-admissible mappings. Inspired from the above mentioned, applied the concept of $\alpha$-admissible mappings to the Darbo theorem. We generalized the Theorem 1.4, in a more general setting. To attain this result, we define $\alpha-\psi$ and $\beta-\psi$ condensing operators and moreover, we used the introduced concepts to propose new fixed point results. We also supply some new coupled fixed point results through a measure of noncompactness for more general class of functions. The obtained results generalize and extend well-known results available in the literature. Moreover, some examples and an application to a functional integral equation are given to illustrate the usability of this idea.

Throughout this paper, we will work in a Banach space $E$ with the norm $\|\cdot\|$ and the zero element $\theta$. Denote by $B(x, r)$ the closed ball centered at $x$ with radius $r$. We use the standard notation $\lambda X$ and $X+Y$ to denote the algebraic operations on sets. Moreover, the symbol $\bar{X}$ stands for the closure of a set $X$ while $\operatorname{co} X$ and $\overline{c o} X$ denotes the convex hull and closed convex hull of $X$ respectively. Finally, we denote $\mathfrak{M}_{E}$ by the family of all nonempty bounded subsets of the space $E$ and by $\mathfrak{N}_{E}$ its subfamily consisting of all relatively compact subsets of $E$.

The remaining part of the paper is organized as follows. First, we recall some known definitions and basic tools which are useful to prove our main results with the corresponding references. In Section 2, we give our proposed fixed point results and their implementation to obtain a coupled fixed point results and in solving a functional integral equation. We begin by taking into account the axiomatic definition of the degree of noncompactness.

Definition 1.1. [4] A mapping $\mu: \mathfrak{M}_{E} \longrightarrow \mathbb{R}_{+}=[0,+\infty)$ is said to be a measure of noncompactness in $E$ if it satisfies the following conditions:

MNC1. The family $k e r \mu=\left\{X \in \mathfrak{M}_{E}: \mu(X)=0\right\}$ is a nonempty set and ker $\mu \subset \mathfrak{N}_{E}$;

MNC2. $X \subseteq Y \Rightarrow \mu(X) \leq \mu(Y)$;

MNC3. $\mu(X)=\mu(\bar{X})$;

MNC4. $\mu(\operatorname{coX})=\mu(X)$;

MNC5. $\mu(\lambda X+(1-\lambda) Y) \leq \lambda \mu(X)+(1-\lambda) \mu(Y)$, for all $\lambda \in[0,1]$;

MNC6. If $X_{n}$ is a sequence of closed sets from $\mathfrak{M}_{E}$ such that $X_{n+1} \subset X_{n}$ for $n=1,2, \cdots$, and if $\lim _{n \rightarrow \infty} \mu\left(X_{n}\right)=$ 0 then $X_{\infty}=\bigcap_{n=1}^{\infty} X_{n} \neq \phi$.

It follows from Definition 1.1, that $X_{\infty}$ is a member of the family $\operatorname{Ker} \mu$. In view that $\mu\left(X_{\infty}\right) \leq \mu\left(X_{n}\right)$ for any $n$, we can deduce that $\mu\left(X_{\infty}\right)=0$. This yields that $X_{\infty} \in k e r \mu$.

Definition 1.2. (Compact operator)[28] An operator $T: X \rightarrow Y$ is referred to as compact if $T(S)$ is relatively compact in a Banach space $Y$ for any bounded subset $S$ in a Banach space $X$.

Theorem 1.3. (Schauder's fixed point theorem) [3] Let C be a nonempty, bounded, closed and convex subset of a Banach space $E$. Then each continuous and compact map $T: C \rightarrow C$ has at least one fixed point in $C$.

Theorem 1.4. (Darbo's fixed point theorem) [29] Let $C$ be a nonempty, bounded, closed and convex subset of a Banach space $E$ and let $T: C \longrightarrow C$ is a continuous mapping such that there exists a constant $k \in[0,1)$ such that

$$
\mu(T S) \leq k \mu(S)
$$


for any nonempty subset $S$ of $C$. Then $T$ has a fixed point in the set $C$.

Denote with $\Psi$ the family of nondecreasing functions $\psi:[0,+\infty) \rightarrow[0,+\infty)$ such that $\lim _{n \rightarrow \infty} \psi^{n}(t)=0$, for each $t>0$, where $\psi^{n}$ is the $n$-th iteration of $\psi$.

Lemma 1.5. For every function $\psi \in \Psi$ the following holds:

if $\psi$ is nondecreasing, then for each $t>0, \lim _{n \rightarrow \infty} \psi^{n}(t)=0$ implies $\psi(t)<t$.

Definition 1.6. [9] Let $T: X \rightarrow X$ and $\alpha: X \times X \rightarrow[0,+\infty)$. We say that $T$ is $\alpha$-admissible if for every $x, y \in X$

$$
\alpha(x, y) \geq 1 \Rightarrow \alpha(T x, T y) \geq 1 .
$$

Example 1.7. [9] Let $X=[0,+\infty)$. Define $T: X \rightarrow X$ by $T x=\ln x, \forall x \in X$, and $\alpha: X \times X \rightarrow[0,+\infty)$ defined by

$$
\alpha(x, y)=\left\{\begin{array}{lll}
2 & \text { if } & x \geq y, \\
0 & \text { if } & x<y
\end{array}\right.
$$

Then $T$ is $\alpha$-admissible.

\section{Main results}

Definition 2.1. ( $\alpha-\psi$ condensing operator) Let $E$ be a Banach space and let $T: E \rightarrow E$ be a given operator. We say that $T$ is an $\alpha-\psi$ condensing operator if there exist two functions $\alpha: E \times E \rightarrow[0,+\infty)$ and $\psi \in \Psi$ such that

$$
\alpha(x, T x) \mu(T X) \leq \psi(\mu(X)),
$$

for any bounded subset $X$ of $E$ and $x \in X$ with $\mu$ an arbitrary measure of noncompactness.

Theorem 2.2. Let $C$ be a nonempty, bounded, closed and convex subset of a Banach space $E$ and there exists $\alpha: E \times E \rightarrow[0,+\infty)$ such that $T: C \rightarrow C$ is a continuous, $\alpha$-admissible and $\alpha-\psi$ condensing operator satisfying the following:

(i) there exist closed and convex $X_{0} \subseteq C$ and $x_{0} \in X_{0}$ such that

$$
T X_{0} \subseteq X_{0}, \alpha\left(x_{0}, T x_{0}\right) \geq 1,
$$

where $\mu$ be an arbitrary measure of noncompactness and $\psi \in \Psi$. Then $T$ has at least one fixed point in the set $C$.

Proof. Firstly, define the sequence of the sets $\left\{X_{n}\right\}$ and elements $\left\{x_{n}\right\}$ as follows:

$$
X_{n}=\overline{C O}\left(T X_{n-1}\right), \quad x_{n}=T x_{n-1} \quad \forall n \geq 1 .
$$

Since $T X_{0} \subseteq X_{0}$, thus

$$
\begin{gathered}
X_{1}=\overline{c o}\left(T X_{0}\right) \subseteq X_{0}, \\
X_{2}=\overline{c o}\left(T X_{1}\right) \subseteq \overline{c o}\left(T X_{0}\right)=X_{1} .
\end{gathered}
$$

Therefore, by continuing this process we obtain $X_{0} \supseteq X_{1} \supseteq X_{2} \supseteq \cdots X_{n} \supseteq X_{n+1} \supseteq \cdots$, and also

$$
T X_{n} \subseteq T X_{n-1} \subseteq \overline{c o}\left(T X_{n-1}\right)=X_{n} .
$$

If there exists an integer $N \geq 0$ such that $\mu\left(X_{N}\right)=0$, then $X_{N}$ is a relatively compact set and also $T X_{N} \subseteq X_{N}$. Thus, Theorem 1.3, implies that $T$ has fixed point. Next, we assume that $\mu\left(X_{n}\right) \neq 0$ for any $n \geq 0$. From equation (2.1) we have $\alpha\left(x_{0}, x_{1}\right)=\alpha\left(x_{0}, T x_{0}\right) \geq 1$, and also $T$ is a $\alpha$-admissible operator implies that $\alpha\left(x_{1}, x_{2}\right) \geq 1$. Recursively, we get the following inequality

$$
\alpha\left(x_{n}, x_{n+1}\right) \geq 1, \forall n \geq 0 .
$$


Furthermore, from our assumptions and equation (2.2), we have

$$
\begin{aligned}
\mu\left(X_{n+1}\right) & \leq \alpha\left(x_{n}, x_{n+1}\right) \mu\left(X_{n+1}\right) \\
& =\alpha\left(x_{n}, T x_{n}\right) \mu\left(\overline{c o}\left(T X_{n}\right)\right) \\
& =\alpha\left(x_{n}, T x_{n}\right) \mu\left(T X_{n}\right) \\
& \leq \psi\left(\mu\left(X_{n}\right)\right),
\end{aligned}
$$

continuing in this manner, we reach the following inequality

$$
\mu\left(X_{n+1}\right) \leq \psi^{n}\left(\mu\left(X_{0}\right)\right) .
$$

Thus, equation (2.3) implies that $\mu\left(X_{n}\right) \rightarrow 0$ as $n \rightarrow \infty$. Since the sequence $\left\{X_{n}\right\}$ is nested so from Definition 1.1 (axiom MNC6), we deduce that the set $X_{\infty}=\bigcap_{n=1}^{\infty} X_{n}$ is nonempty, closed and convex subset of the set $X_{0}$. On the other hand, $\mu\left(X_{\infty}\right) \leq \mu\left(X_{n}\right), \forall n \in N$ implies that $\mu\left(X_{\infty}\right)=0$. Hence we get that $X_{\infty}$ is a member of the ker $\mu$, which implies $X_{\infty}$ is compact. Moreover, we have $X_{\infty} \subset X_{n}$ and $T\left(X_{n}\right) \subset X_{n}$ for all $n \in N$. Therefore, $T: X_{\infty} \rightarrow X_{\infty}$ is well defined. For any bounded $A \subset X_{\infty}$, we have $T(A) \subset X_{\infty}$ and $\overline{T(A)}$ is a compact subset of $X_{\infty}$, implies that $T$ is compact operator. Therefore, Theorem 1.3, completes the proof.

Remark 2.3. In Theorem 2.2, we get Darbo's theorem if we take $\alpha(x, y)=1$ and $\psi(t)=k t$ for all $t \geq 0$ and for $k \in[0,1)$.

Now using the above theorem, we prove the following corollary which belongs to the classical metric fixed point theory.

Corollary 2.4. Let $C$ be a nonempty, bounded, closed and convex subset of a Banach space $E$ and there exists $\alpha: E \times E \rightarrow[0,+\infty)$ such that $T: C \rightarrow C$ is a continuous and $\alpha$-admissible operator satisfying the following:

(i) for any $x, y, u \in X$, we have

$$
\alpha(u, T u)\|T x-T y\| \leq \psi(\|x-y\|) ;
$$

(ii) there exist closed and convex $X_{0} \subseteq C$ and $x_{0} \in X_{0}$ such that

$$
T X_{0} \subseteq X_{0}, \alpha\left(x_{0}, T x_{0}\right) \geq 1,
$$

where $\psi \in \Psi$. Then $T$ has at least one fixed point in $C$.

Proof. Let $\mu: \mathfrak{M}_{E} \rightarrow \mathbb{R}_{+}$be defined as

$$
\mu(X)=\operatorname{diam} X,
$$

where $\operatorname{diam} X=\sup \{\|x-y\|: x, y \in X\}$ stands for the diameter of $X$. It is easy to see that $\mu$ is a measure of noncompactness in a space $E$ in the sense of Definition 1.1. Furthermore, from equation (2.4) and $\psi$ being nondecreasing we have

$$
\alpha(u, T u) \sup _{x, y \in X}\|T x-T y\| \leq \psi\left(\sup _{x, y \in X}\|x-y\|\right),
$$

which implies that

$$
\alpha(u, T u) \mu(T X) \leq \psi(\mu(X)),
$$

so from Theorem 2.2, we get the desired result.

Proposition 2.5. If $\alpha(x, x) \geq 1$ for all $x \in E$, then the set of all fixed points of $T$ in Theorem 2.2, is a compact set.

Proof. Let $F=\{x \in C: T x=x\}$ be the set of all fixed points of $T$ and $\mu(F) \neq 0$, then by $\alpha-\psi$ condensing operator of $T$ we have

$$
\alpha(x, T x) \mu(T F) \leq \psi(\mu(F))<\mu(F),
$$


which is a contradiction from above inequality since $T(F)=F$. This implies that $F$ is a relatively compact set. Now taking into account any convergent sequence $\left\{x_{n}\right\} \subset F$ and $x_{n} \rightarrow x^{\star}$, we have $x^{\star} \in C$, because $C$ is closed. The continuity of $T$ implies that $x_{n}=T x_{n} \rightarrow T x^{\star}$ and $T x^{\star}=x^{\star}$, which means that $x^{\star} \in F$, i.e. $F$ is a compact set.

Example 2.6. The operator $T: B C\left(\mathbb{R}_{+}\right) \rightarrow B C\left(\mathbb{R}_{+}\right)$defined by

$$
T x(t)=\left\{\begin{array}{lll}
\frac{x}{2} & \text { if } & \|x\| \leq 1, \\
2 x-2 & \text { if } & \|x\|>1,
\end{array}\right.
$$

and let $B C\left(\mathbb{R}_{+}\right)$denote the space of all real-valued bounded and continuous functions on $\mathbb{R}_{+}$. First, we observe that Theorem 1.4, cannot be applied in the case when $\|x\|,\|y\|>1$ and we obtain

$$
\|T x(t)-T y(t)\|=\|2 x(t)-2-2 y(t)+2\|=2\|x(t)-y(t)\|,
$$

and by taking supremum value on both sides we have

$$
\mu(T X)=2 \mu(X) .
$$

Now, we define the mapping $\alpha: B C\left(\mathbb{R}_{+}\right) \times B C\left(\mathbb{R}_{+}\right) \longrightarrow B C\left(\mathbb{R}_{+}\right)$by

$$
\alpha(x, y)= \begin{cases}1 & \|x\| \leq 1, \\ 0 & \text { otherwise. }\end{cases}
$$

Clearly, T is a $\alpha-\psi$ condensing operator with $\psi(t)=\frac{t}{2}$ for $t \geq 0$, and $\mu(X)=\operatorname{diam} X$.

In order to prove the next results, we need the following definitions.

Definition 2.7. ( $\beta$-admissible) Let $T: E \rightarrow E$ and $\beta: 2^{E} \rightarrow[0,+\infty)$. We say that $T$ is $\beta$-admissible operator if for every $X \in 2^{E}$, we have

$$
\beta(X) \geq 1 \Rightarrow \beta(\overline{c o} T X) \geq 1 .
$$

Example 2.8. Let $T: B C\left(\mathbb{R}_{+}\right) \rightarrow B C\left(\mathbb{R}_{+}\right)$be defined by $T x(t)=\lambda x(t)$ for $\lambda \geq 1$, and also there exist $\beta$ : $2^{B C\left(\mathbb{R}_{+}\right)} \rightarrow \mathbb{R}_{+}$such that

$$
\beta(X)=\operatorname{diam}(X)
$$

for every $X \subset B C\left(\mathbb{R}_{+}\right)$. Then $T$ is $\beta$-admissible operator.

Example 2.9. Let $T: B C\left(\mathbb{R}_{+}\right) \rightarrow B C\left(\mathbb{R}_{+}\right)$and there exist $\beta: 2^{B C\left(\mathbb{R}_{+}\right)} \rightarrow \mathbb{R}_{+}$such that

$$
T x(t)=e^{x(t)} \text { and } \beta(X)=\sup \{\|x\|: x \in X\}
$$

for every $x \in B C\left(\mathbb{R}_{+}\right), X \subset B C\left(\mathbb{R}_{+}\right)$respectively. Then $T$ is $\beta$-admissible operator.

Definition 2.10. ( $\beta-\psi$ condensing operator) Let $E$ be a Banach space and let $T: E \rightarrow E$ be a given operator. We say that $T$ is $\beta$ - $\psi$ condensing operator if there exist two functions $\beta: 2^{E} \rightarrow[0,+\infty)$ and $\psi \in \Psi$ such that

$$
\beta(X) \mu(T X) \leq \psi(\mu(X)),
$$

for any bounded subset $X$ of $E$ with $\mu$ an arbitrary measure of noncompactness.

Theorem 2.11. Let $C$ be a nonempty, bounded, closed and convex subset of a Banach space $E$ and there exists $\beta: 2^{E} \rightarrow[0,+\infty)$ such that $T: C \rightarrow C$ is a continuous, $\beta$-admissible and $\beta$ - $\psi$ condensing operator satisfying the following:

(i) there exist closed and convex $X_{0} \subseteq C$ such that

$$
T X_{0} \subseteq X_{0}, \beta\left(X_{0}\right) \geq 1,
$$


where $\mu$ be an arbitrary measure of noncompactness and $\psi \in \Psi$. Then $T$ has at least one fixed point in $C$.

Proof. Similarly, as in the proof of Theorem 2.2, we define the following sequence:

$$
X_{n}=\overline{c o}\left(T X_{n-1}\right) .
$$

Since $T X_{0} \subseteq X_{0}$, thus

$$
\begin{gathered}
X_{1}=\overline{c o}\left(T X_{0}\right) \subseteq X_{0}, \\
X_{2}=\overline{c o}\left(T X_{1}\right) \subseteq \overline{c o}\left(T X_{0}\right)=X_{1} .
\end{gathered}
$$

Therefore, by continuing this process we obtain

$$
X_{0} \supseteq X_{1} \supseteq X_{2} \supseteq \cdots X_{n} \supseteq X_{n+1} \supseteq \cdots
$$

and

$$
T X_{n} \subseteq T X_{n-1} \subseteq \overline{c o}\left(T X_{n-1}\right)=X_{n} .
$$

If there exists an integer $N_{0} \geq 0$ such that $\mu\left(X_{N_{0}}\right)=0$, implies $X_{N_{0}}$ is relatively compact and also $T X_{N_{0}} \subseteq X_{N_{0}}$. Thus, Theorem 1.3, implies that $T$ has fixed point. Moreover, we assume that $\mu\left(X_{n}\right) \neq 0$ for all $n \geq 0$. For $T$ to be a $\beta$-admissible operator and from equation (2.7), we obtain $\beta\left(X_{1}\right)=\beta\left(\overline{c o} T X_{0}\right) \geq 1$. Recursively, we obtain the following inequality

$$
\beta\left(X_{n}\right) \geq 1, \forall n \geq 0 .
$$

Furthermore, from equation (2.8) we have

$$
\begin{aligned}
\mu\left(X_{n+1}\right) & \leq \beta\left(X_{n}\right) \mu\left(X_{n+1}\right) \\
& =\beta\left(X_{n}\right) \mu\left(\overline{c o}\left(T X_{n}\right)\right) \\
& =\beta\left(X_{n}\right) \mu\left(T X_{n}\right) \\
& \leq \psi\left(\mu\left(X_{n}\right)\right),
\end{aligned}
$$

continuing in this manner, we reach at the following inequality

$$
\mu\left(X_{n+1}\right) \leq \psi^{n}\left(\mu\left(X_{0}\right)\right) .
$$

Equation (2.9) implies that $\mu\left(X_{n}\right) \rightarrow 0$ as $n \rightarrow \infty$. Since the sequence $\left\{X_{n}\right\}$ is nested and in view of Definition 1.1(axiom MNC6), we deduce that the set $X_{\infty}=\bigcap_{n=1}^{\infty} X_{n}$ is nonempty, closed and convex subset of the set $X_{0}$. Hence, we get that $X_{\infty}$ is a member of the ker $\mu$ and $T$ maps $X_{\infty}$ into itself and taking into account Theorem 1.3 , gives the desired result.

Remark 2.12. From Theorem 2.11, we get Darbo's theorem if we take $\beta(X)=1$ and $\psi(t)=k t$ for all $t \geq 0$ and for some $k \in[0,1)$.

Now let us pay attention to the following corollary from the above theorem which belongs to the classical metric fixed point theory.

Corollary 2.13. Let $C$ be a nonempty, bounded, closed and convex subset of a Banach space $E$ and there exists $\beta: 2^{E} \rightarrow[0,+\infty)$ such that $T: C \rightarrow C$ is a continuous and $\beta$-admissible operator satisfying the following:

(i) for any $X \in \mathfrak{M}_{C}$ and $x, y \in X$, we have

$$
\beta(X)\|T x-T y\| \leq \psi(\|x-y\|) ;
$$

(ii) there exist closed and convex $X_{0} \subseteq C$ such that

$$
T X_{0} \subseteq X_{0}, \beta\left(X_{0}\right) \geq 1
$$

where $\psi \in \Psi$. Then $T$ has at least one fixed point in the set $C$. 
Proof. Let $\mu: \mathfrak{M}_{E} \rightarrow \mathbb{R}_{+}$be defined as:

$$
\mu(X)=\operatorname{diam} X
$$

where $\operatorname{diam} X=\sup \{\|x-y\|: x, y \in X\}$ stands for the diameter of $X$. Clearly, from Definition 1.1, $\mu$ is a measure of noncompactness in the space $E$. Further, from (2.10) and $\psi$ to be nondecreasing we have

$$
\beta(X) \sup _{x, y \in X}\|T x-T y\| \leq \psi\left(\sup _{x, y \in X}\|x-y\|\right),
$$

implies that

$$
\beta(X) \mu(T X) \leq \psi(\mu(X)) .
$$

So from Theorem 2.11, we get desired result.

Proposition 2.14. If $\beta(X) \geq 1$ for all $X \in 2^{E}$ having $T(X)=X$, then the set of all fixed points of $T$ in Theorem 2.11, is a compact set.

Proof. Let $F=\{x \in C: T x=x\}$ be the set of all fixed points of $T$ and $\mu(F) \neq 0$, from the assumption of Theorem 2.11, we have

$$
\beta(F) \mu(T F) \leq \psi(\mu(F))<\mu(F),
$$

which is a contradiction from above inequality since $T(F)=F$. This implies that $F$ is a relatively compact set. Now taking into account any convergent sequence $\left\{x_{n}\right\} \subset F$ and $x_{n} \rightarrow x^{\star}$, we have $x^{\star} \in C$, because $C$ is closed. The continuity of $T$ implies that $x_{n}=T x_{n} \rightarrow T x^{\star}$ and $T x^{\star}=x^{\star}$, which means that $x^{\star} \in F$, i.e. $F$ is a compact set.

Example 2.15. The operator $T: B C\left(\mathbb{R}_{+}\right) \rightarrow B C\left(\mathbb{R}_{+}\right)$defined by

$$
T x(t)=\left\{\begin{array}{lll}
\frac{x}{2} & \text { if } & \|x\| \leq 1, \\
2 x-\frac{3}{2} & \text { if } & \|x\|>1
\end{array}\right.
$$

At first we take a look at that Theorem 1.4, cannot be carried out in the case while $\|x\|,\|y\|>1$ and we obtain

$$
\|T x(t)-T y(t)\|=\left\|2 x(t)-\frac{3}{2}-2 y(t)+\frac{3}{2}\right\|=2\|x(t)-y(t)\| .
$$

Through taking supremum value on both sides we have

$$
\mu(T X)=2 \mu(X) .
$$

However, if we define $\beta: 2^{B C\left(\mathbb{R}_{+}\right)} \rightarrow[0,+\infty)$ by

$$
\beta(X)= \begin{cases}1 & \text { if }\|x\| \leq 1, \forall x \in X, \\ 0 & \text { otherwise. }\end{cases}
$$

Certainly $\mathrm{T}$ is an $\beta-\psi$ condensing operator with $\psi(t)=\frac{t}{2}$ for $t \geq 0$, and $\mu(X)=\operatorname{diam} X$.

\section{Coupled fixed point theorems}

In this section, we prove some coupled fixed point theorems using $\alpha$-admissible and $\beta$-admissible operators. Before that let us take into account some basic definitions about coupled fixed points and the measure of noncompactness in product spaces.

Definition 2.16. (Coupled fixed point)[10] An element $(x, y)$ in $E^{2}$ is called a coupled fixed point of a mapping $T: E^{2} \rightarrow E$ if $T(x, y)=x$ and $T(y, x)=y$. 
Lemma 2.17. [30] Suppose that $\mu_{1}, \mu_{2}, \ldots, \mu_{n}$ are the measures of noncompactness in Banach spaces $E_{1}, E_{2}, \cdots, E_{n}$ respectively. Moreover, assume that the function $F:[0, \infty)^{n} \longrightarrow[0, \infty)$ is convex and $F\left(x_{1}, x_{2}, \cdots, x_{n}\right)=0$ if and only if each $x_{i}=0$ for all $i=1,2, \cdots, n$. Then we define a measure of noncompactness on $E_{1} \times E_{2} \times, \cdots, \times E_{n}$ as follows:

$$
\mu(S)=F\left(\mu_{1}\left(S_{1}\right), \mu_{2}\left(S_{2}\right), \ldots, \mu_{n}\left(S_{n}\right)\right),
$$

where $S_{i}$ denotes the natural projections of $S$ into $E_{i}$ for $i=1,2, \cdots, n$.

Additionally, as a result of Lemma 2.17, we present the following examples.

Example 2.18. Let $\mu$ be a measure of noncompactness on a Banach space $E$, and let the function $F:[0,+\infty)^{2} \rightarrow[0,+\infty)$ be convex with $F\left(x_{1}, x_{2}\right)=0$ if and only if $x_{i}=0$ for $i=1,2$. Then

$$
\mu^{\star}(X)=F\left(\mu\left(X_{1}\right), \mu\left(X_{2}\right)\right),
$$

defines a measure of noncompactness in $E \times E$, where $X_{i}$ denote the natural projections of $X$ into $E$.

Example 2.19. Let $\mu$ be a measure of noncompactness on a Banach space $E$, considering $F(x, y)=x+y$ for any $(x, y) \in[0,+\infty)^{2}$. Then we see that $F$ is convex and $F(x, y)=0$ if and only if $x=y=0$, hence all the conditions of Lemma 2.17, are satisfied. Therefore, $\mu^{*}(X)=\mu\left(X_{1}\right)+\mu\left(X_{2}\right)$ defines a measure of noncompactness in the space $E \times E$ where $X_{i}, i=1,2$ denote the natural projections of $X$ into $E$.

Example 2.20. Let $\mu$ be a measure of noncompactness on a Banach space $E$. If we define $F(x, y)=\max \{x, y\}$ for any $(x, y) \in[0,+\infty)^{2}$, then all the conditions of Lemma 2.17, are satisfied and $\mu^{\star}(X)=\max \left\{\mu\left(X_{1}\right), \mu\left(X_{2}\right)\right\}$ is a measure of noncompactness in the space $E \times E$ where $X_{i}, i=1,2$ denote the natural projections of $X$ into E.

Theorem 2.21. Let $C$ be a nonempty, bounded, closed and convex subset of a Banach space $E$ and $\gamma: E^{2} \times E^{2} \rightarrow$ $[0,+\infty)$, let $T: C \times C \rightarrow C$ be continuous and also fulfilling the following conditions:

(i) let $X_{1} \times X_{2} \subseteq C \times C$ and $T\left(x_{1}, y_{1}\right)=x_{2}, T\left(y_{1}, x_{1}\right)=y_{2}$ such that

$$
\gamma\left(\left(x_{1}, y_{1}\right),\left(x_{2}, y_{2}\right)\right) \mu\left(T\left(X_{1} \times X_{2}\right)\right) \leq \frac{1}{2} \psi\left(\mu\left(X_{1}\right)+\mu\left(X_{2}\right)\right),
$$

for any $\left(x_{1}, y_{1}\right) \in X_{1} \times X_{2}$;

(ii) for all $(x, y),(u, v) \in C \times C$ and $\gamma((x, y),(u, v)) \geq 1$ we have

$$
\gamma((T(x, y), T(y, x)),(T(u, v), T(v, u))) \geq 1 ;
$$

(iii) further, there exist closed and convex $X_{0}, Y_{0} \subseteq C$ such that $T\left(X_{0} \times Y_{0}\right) \subseteq X_{0}, T\left(Y_{0} \times X_{0}\right) \subseteq Y_{0}$ and also there exists $\left(x_{0}, y_{0}\right) \in X_{0} \times Y_{0}$ such that

$$
\gamma\left(\left(x_{0}, y_{0}\right),\left(T\left(x_{0}, y_{0}\right), T\left(y_{0}, x_{0}\right)\right)\right) \geq 1,
$$

and

$$
\gamma\left(\left(y_{0}, x_{0}\right),\left(T\left(y_{0}, x_{0}\right), T\left(x_{0}, y_{0}\right)\right)\right) \geq 1,
$$

where $\mu$ be an arbitrary measure of noncompactness on $E$ and $\psi \in \Psi$. Then $T$ has at least one coupled fixed point in $C \times C$.

Proof. To prove this theorem, we need to define $G: C \times C \rightarrow C \times C$ by

$$
G(x, y)=(T(x, y), T(y, x)) .
$$

From Example 2.19, we take $\mu^{*}$ is a measure of noncompactness on $E^{2}$ as follows:

$$
\mu^{\star}(X)=\mu\left(X_{1}\right)+\mu\left(X_{2}\right),
$$


where $X_{1}$ and $X_{2}$ are the natural projections of $X$ on $E$. Further, we define $\alpha: X^{2} \times X^{2} \rightarrow[0,+\infty)$ as follows:

$$
\alpha\left(\left(x_{1}, y_{1}\right),\left(x_{2}, y_{2}\right)\right)=\min \left\{\gamma\left(\left(x_{1}, y_{1}\right),\left(x_{2}, y_{2}\right)\right), \gamma\left(\left(y_{1}, x_{1}\right),\left(y_{2}, x_{2}\right)\right)\right\} .
$$

We need to show that $G$ satisfies all the conditions of Theorem 2.2, then $G$ has a fixed point in $C \times C$, which is the coupled fixed point of the operator $T$. We list the following conditions which we want to meet for the desired result:

- $G$ is continuous and $\alpha$-admissible operator;

- $\alpha\left(\left(x_{1}, y_{1}\right), G\left(x_{1}, y_{1}\right)\right) \mu^{*}(G(X)) \leq \psi\left(\mu^{*}(X)\right)$, for $\left(x_{1}, y_{1}\right) \in X$;

- There exists closed and convex subset $A_{0} \subseteq C \times C$, and $\left(x_{0}, y_{0}\right) \in A_{0}$ such that

$$
G A_{0} \subseteq A_{0}, \alpha\left(\left(x_{0}, y_{0}\right), G\left(x_{0}, y_{0}\right)\right) \geq 1 .
$$

We can easily see from equation (2.16) that $G$ is continuous. Now from equations (2.13) and (2.16), it is clear that whenever $\alpha\left(\left(x_{1}, y_{1}\right),\left(x_{2}, y_{2}\right)\right) \geq 1$, we have $\alpha\left(G\left(x_{1}, y_{1}\right), G\left(x_{2}, y_{2}\right)\right) \geq 1$, which shows that $G$ is $\alpha$-admissible. Also, from equation (2.12) we have

$$
\begin{aligned}
& \gamma\left(\left(x_{1}, y_{1}\right),\left(x_{2}, y_{2}\right)\right) \mu\left(T\left(X_{1} \times X_{2}\right)\right) \leq \frac{1}{2} \psi\left(\mu\left(X_{1}\right)+\mu\left(X_{2}\right)\right), \\
& \gamma\left(\left(y_{1}, x_{1}\right),\left(y_{2}, x_{2}\right)\right) \mu\left(T\left(X_{2} \times X_{1}\right)\right) \leq \frac{1}{2} \psi\left(\mu\left(X_{2}\right)+\mu\left(X_{1}\right)\right) .
\end{aligned}
$$

From equations (2.17), (2.18) and (2.19), we obtain

$$
\alpha\left(\left(x_{1}, y_{1}\right),\left(x_{2}, y_{2}\right)\right)\left(\mu\left(T\left(X_{1} \times X_{2}\right)\right)+\mu\left(T\left(X_{2} \times X_{1}\right)\right)\right) \leq \psi\left(\mu^{\star}\left(X_{1} \times X_{2}\right)\right) .
$$

Eventually, from equations (2.16) and (2.17) we have

$$
\alpha\left(\left(x_{1}, y_{1}\right),\left(x_{2}, y_{2}\right)\right) \mu^{\star}(G(X)) \leq \psi\left(\mu^{\star}(X)\right) .
$$

Now allow taking $A_{0}=X_{0} \times Y_{0}$ and from equations (2.14) and (2.15) we have $G\left(A_{0}\right) \subseteq A_{0}$ and $\alpha\left(\left(x_{0}, y_{0}\right), G\left(x_{0}, y_{0}\right)\right) \geq 1$. So all the conditions of the Theorem 2.2 are satisfied and $G$ has a fixed point in $C \times C$.

Corollary 2.22. Let $C$ be a nonempty, bounded, closed and convex subset of a Banach space $E$ and $\gamma: E^{2} \times E^{2} \rightarrow$ $[0,+\infty)$, let $T: C \times C \rightarrow C$ be continuous and also satisfying the following conditions:

(i) let $X_{1} \times X_{2} \subseteq C \times C$ and $T\left(x_{1}, y_{1}\right)=x_{2}, T\left(y_{1}, x_{1}\right)=y_{2}$ such that

$$
\gamma\left(\left(x_{1}, y_{1}\right),\left(x_{2}, y_{2}\right)\right) \mu\left(T\left(X_{1} \times X_{2}\right)\right) \leq \frac{1}{2} \psi\left(\max \left\{\mu\left(X_{1}\right), \mu\left(X_{2}\right)\right\}\right),
$$

for any $\left(x_{1}, y_{1}\right) \in X_{1} \times X_{2}$;

(ii) for all $(x, y),(u, v) \in C \times C$ and $\gamma((x, y),(u, v)) \geq 1$ we have

$$
\gamma((T(x, y), T(y, x)),(T(u, v), T(v, u))) \geq 1 ;
$$

(iii) also, there exist closed and convex $X_{0}, Y_{0} \subseteq C$ such that $T\left(X_{0} \times Y_{0}\right) \subseteq X_{0}, T\left(Y_{0} \times X_{0}\right) \subseteq Y_{0}$ and also there exists $\left(x_{0}, y_{0}\right) \in X_{0} \times Y_{0}$ such that

$$
\gamma\left(\left(x_{0}, y_{0}\right),\left(T\left(x_{0}, y_{0}\right), T\left(y_{0}, x_{0}\right)\right)\right) \geq 1,
$$

and

$$
\gamma\left(\left(y_{0}, x_{0}\right),\left(T\left(y_{0}, x_{0}\right), T\left(x_{0}, y_{0}\right)\right)\right) \geq 1,
$$

where $\mu$ be an arbitrary measure of noncompactness on $E$ and $\psi \in \Psi$. Then $T$ has at least one coupled fixed point in $C \times C$. 
Theorem 2.23. Let $C$ be a nonempty, bounded, closed and convex subset of a Banach space $E$ and $\gamma: 2^{E_{1} \times E_{2}} \rightarrow$ $[0,+\infty)$, let $T: C \times C \rightarrow C$ be continuous and satisfying the following conditions:

(i) for any $X_{1} \times X_{2} \subseteq C \times C$, we have

$$
\gamma\left(X_{1} \times X_{2}\right) \mu\left(T\left(X_{1} \times X_{2}\right)\right) \leq \frac{1}{2} \psi\left(\mu\left(X_{1}\right)+\mu\left(X_{2}\right)\right) ;
$$

(ii) for any $U \times V \subseteq C \times C$ and $\gamma(U \times V) \geq 1$, we have

$$
\gamma(\overline{c o}(T(U \times V) \times T(V \times U))) \geq 1 ;
$$

(iii) there exist closed and convex $X_{0}, Y_{0} \subseteq C$ such that $T\left(X_{0} \times Y_{0}\right) \subseteq X_{0}$ and $T\left(Y_{0} \times X_{0}\right) \subseteq Y_{0}$ such that

$$
\gamma\left(X_{0} \times Y_{0}\right) \geq 1 \text { and } \gamma\left(Y_{0} \times X_{0}\right) \geq 1
$$

where $\mu$ be an arbitrary measure of noncompactness and $\psi \in \Psi$. Then T has at least one coupled fixed point in $C \times C$.

Proof. Firstly, we define $G: C \times C \rightarrow C \times C$ such that

$$
G(x, y)=(T(x, y), T(y, x)),
$$

also take $\mu^{\star}$ is measure of noncompactness on $E^{2}$ as follows:

$$
\mu^{\star}(X)=\mu\left(X_{1}\right)+\mu\left(X_{2}\right),
$$

where $X_{1}$ and $X_{2}$ are the natural projections of $X$ on $E$. Further, we define $\beta: 2^{E_{1} \times E_{2}} \rightarrow[0,+\infty)$ in the following way:

$$
\beta(X)=\min \left\{\gamma\left(X_{1} \times X_{2}\right), \gamma\left(X_{2} \times X_{1}\right)\right\},
$$

where $X_{1}$ and $X_{2}$ are the natural projections of $X$ on $E$. To get required result we need to show that $G$ satisfies all the conditions of Theorem 2.11, which are following:

- $G$ is continuous and $\beta$-admissible operator;

- $\beta(X) \mu^{\star}(G(X)) \leq \psi\left(\mu^{\star}(X)\right)$;

- There exists closed and convex subset $A_{0} \subseteq C \times C$ such that $G A_{0} \subseteq A_{0}$ and $\beta\left(A_{0}\right) \geq 1$.

Clearly $G$ is continuous and also whenever $\beta\left(X_{1} \times X_{2}\right) \geq 1$, we have $\beta\left(\overline{c o} G\left(X_{1} \times X_{2}\right)\right) \geq 1$, which shows that $G$ is $\beta$-admissible. From our hypothesis we have

$$
\begin{aligned}
& \gamma\left(X_{1} \times X_{2}\right) \mu\left(T\left(X_{1} \times X_{2}\right)\right) \leq \frac{1}{2} \psi\left(\mu\left(X_{1}\right)+\mu\left(X_{2}\right)\right), \\
& \gamma\left(X_{2} \times X_{1}\right) \mu\left(T\left(X_{2} \times X_{1}\right)\right) \leq \frac{1}{2} \psi\left(\mu\left(X_{2}\right)+\mu\left(X_{1}\right)\right) .
\end{aligned}
$$

From equations (2.28), (2.29) and (2.30) we obtain

$$
\beta(X)\left(\mu\left(T\left(X_{1} \times X_{2}\right)\right)+\mu\left(T\left(X_{2} \times X_{1}\right)\right)\right) \leq \psi\left(\mu^{*}\left(X_{1} \times X_{2}\right)\right) .
$$

Finally, from equations (2.27) and (2.28), we get the following inequality

$$
\beta(X) \mu^{*}(G(X)) \leq \psi\left(\mu^{*}(X)\right) .
$$

In the end, from equation (2.26) we take $A_{0}=X_{0} \times Y_{0}$ and we have $G\left(A_{0}\right) \subseteq A_{0}$ and also $\beta\left(A_{0}\right) \geq 1$. all the conditions of the Theorem 2.11 are satisfied and $G$ has a fixed point in $C \times C$.

Corollary 2.24. Let $C$ be a nonempty, bounded, closed and convex subset of a Banach space E and $\gamma: 2^{E_{1} \times E_{2}} \rightarrow$ $[0,+\infty)$, let $T: C \times C \rightarrow C$ be continuous and satisfying the following conditions: 
(i) for any $X_{1} \times X_{2} \subseteq C \times C$, we have

$$
\gamma\left(X_{1} \times X_{2}\right) \mu\left(T\left(X_{1} \times X_{2}\right)\right) \leq \frac{1}{2} \psi\left(\max \left\{\mu\left(X_{1}\right), \mu\left(X_{2}\right)\right\}\right) ;
$$

(ii) for any $U \times V \subseteq C \times C$ and $\gamma(U \times V) \geq 1$ we have

$$
\gamma(\overline{c o}(T(U \times V) \times T(V \times U))) \geq 1 ;
$$

(iii) there exist closed and convex $X_{0}, Y_{0} \subseteq C$ such that $T\left(X_{0} \times Y_{0}\right) \subseteq X_{0}$ and $T\left(Y_{0} \times X_{0}\right) \subseteq Y_{0}$ such that

$$
\gamma\left(X_{0} \times Y_{0}\right) \geq 1 \text { and } \gamma\left(Y_{0} \times X_{0}\right) \geq 1 \text {, }
$$

where $\mu$ be an arbitrary measure of noncompactness and $\psi \in \Psi$. Then $T$ has at least one coupled fixed point in $C \times C$.

\section{A functional integral equation}

In this section, we are going to present an application of Theorem 2.11, a study of existence of solution for an integral equation defined on the Banach spaces $B C\left(\mathbb{R}_{+}\right)$, which includes all continuous real-valued and bounded functions on $\mathbb{R}_{+}$and equipped with the norm, i.e.

$$
\|x\|=\sup \{|x(t)|: t>0\} .
$$

The measure of noncompactness on $B C\left(\mathbb{R}_{+}\right)[21,30-32]$ for a positive fixed $t$ on $\mathfrak{M}_{B C\left(\mathbb{R}_{+}\right)}$is defined as follows:

$$
\mu(X)=\omega_{0}(X)+\limsup _{t \rightarrow \infty} \operatorname{diam} X(t),
$$

where $\operatorname{diam} X(t)=\sup \{|x(t)-y(t)|: x, y \in X\}$ and $X(t)=\{x(t): x \in X\}$. Before defining the $\omega_{0}(X)$, we first need to define the modulus of continuity for any $x \in X$ and $\epsilon>0$. The modulus of the continuity of $x$ on the interval $[0, T]$ denoted by $\omega^{T}(x, \epsilon)$, i.e.

$$
\omega^{T}(x, \epsilon)=\sup \{|x(t)-x(s)|: t, s \in[0, T],|t-s| \leq \epsilon\},
$$

where

$$
\begin{gathered}
\omega^{T}(X, \epsilon)=\sup \left\{\omega^{T}(x, \epsilon): x \in X\right\}, \\
\omega_{0}^{T}(X)=\lim _{\epsilon \rightarrow 0} \omega^{T}(X, \epsilon), \\
\omega_{0}(X)=\lim _{T \rightarrow \infty} \omega_{0}^{T}(X) .
\end{gathered}
$$

As an application of the Theorem 2.11, we are going to have a look at the existence of the solution for the following integral equation:

$$
x(t)=A(t)+h(t, x(\xi(t)))+f\left(t, x(\xi(t)), \varphi\left(\int_{0}^{\beta(t)} g(t, s, x(\eta(s))) d s\right)\right) .
$$

For this cause, we assume the following conditions:

i) the function $A: \mathbb{R}_{+} \rightarrow \mathbb{R}_{+}$is continuous and bounded with $M_{1}=\sup \left\{|A(t)|: t \in \mathbb{R}_{+}\right\}$;

ii) $\xi, \eta, \beta: \mathbb{R}_{+} \rightarrow \mathbb{R}_{+}$are continuous functions and $\xi(t) \rightarrow \infty$ as $t \rightarrow \infty$;

iii) the function $\varphi$ is continuous and there exist $\alpha, \delta>0$, such that

$$
\left|\varphi\left(t_{1}\right)-\varphi\left(t_{2}\right)\right| \leq \delta\left|t_{1}-t_{2}\right|^{\alpha},
$$

for any $t_{1}, t_{2} \in \mathbb{R}_{+}$and moreover, $\varphi(0)=0$; 
iv) the functions $h: \mathbb{R}_{+} \times \mathbb{R} \rightarrow \mathbb{R}$ and $f: \mathbb{R}_{+} \times \mathbb{R} \times \mathbb{R} \rightarrow \mathbb{R}$ are continuous and $\psi \in \Psi$, and there exists nondecreasing continuous function $\theta: \mathbb{R}_{+} \rightarrow \mathbb{R}$ with $\theta(0)=0$. Also, there exists $\zeta: R^{2} \rightarrow R$ with $\zeta\left(x_{1}, y_{1}\right) \geq 0$ such that

$$
\left|h\left(t, x_{1}\right)-h\left(t, y_{1}\right)\right| \leq \frac{1}{2} \psi\left(\left|x_{1}-y_{1}\right|\right)
$$

and

$$
\left|f\left(t, x_{1}, d_{1}\right)-f\left(t, y_{1}, d_{2}\right)\right| \leq \frac{1}{2}\left(\psi\left(\left|x_{1}-y_{1}\right|\right)\right)+\theta\left(\left|d_{1}-d_{2}\right|\right),
$$

for all $x_{1}, y_{1} \in \mathbb{R}$ for any $t \geq 0$;

v) the functions defined by $t \rightarrow|h(t, 0)|$ and $t \rightarrow|f(t, 0,0)|$ are bounded on $\mathbb{R}_{+}$; i.e.

$$
\begin{gathered}
M_{2}=\sup \left\{|h(t, 0)|: t \in \mathbb{R}_{+}\right\}<\infty, \\
M_{3}=\sup \left\{|f(t, 0,0)|: t \in \mathbb{R}_{+}\right\}<\infty ;
\end{gathered}
$$

vi) the function $g: \mathbb{R}_{+} \times \mathbb{R}_{+} \times \mathbb{R} \rightarrow \mathbb{R}$ is a continuous function and there exists positive solution $r_{0}$ of the inequality

$$
M_{1}+\psi\left(r_{0}\right)+M_{2}+M_{3}+\theta\left(\delta M_{4}\right)<r_{0},
$$

where $M_{4}$ is a positive constant defined by the following equalities

$$
M_{4}=\sup \left\{\left|\int_{0}^{\beta(t)} g(t, s, x(\eta(s))) d s\right|^{\alpha}: t \in \mathbb{R}_{+} \text {and } x \in B C\left(\mathbb{R}_{+}\right)\right\},
$$

and

$$
\lim _{t \rightarrow \infty} \int_{0}^{\beta(t)}|g(t, s, x(\eta(s)))-g(t, s, u(\eta(s)))| d s=0,
$$

uniformly with respect to $x, u \in B C\left(\mathbb{R}_{+}\right)$;

vii) for $\zeta(x(t), y(t)) \geq 0$, for all $x, y \in X \subseteq B C\left(\mathbb{R}_{+}\right)$and for any $t \in \mathbb{R}_{+}$, implies that $\zeta(u(t), v(t)) \geq 0$, for all $u, v \in \overline{c o} T(X)$ and for any $t \in \mathbb{R}_{+}$. Moreover, $\zeta\left(x_{0}(t), y_{0}(t)\right) \geq 0$ for all $x_{0}, y_{0} \in B_{r_{0}}$ (ball of radius of $r_{0}$ in $B C\left(\mathbb{R}_{+}\right)$), for any $t \in \mathbb{R}_{+}$.

Theorem 2.25. Suppose that (i)-(vii) holds; then the system of integral equation

$$
x(t)=A(t)+h(t, x(\xi(t)))+f\left(t, x(\xi(t)), \varphi\left(\int_{0}^{\beta(t)} g(t, s, x(\eta(s))) d s\right)\right)
$$

has at least one solution in the space $B C\left(\mathbb{R}_{+}\right)$.

Proof. Let $T: B C\left(\mathbb{R}_{+}\right) \rightarrow B C\left(\mathbb{R}_{+}\right)$be an operator defined by

$$
(T x)(t)=A(t)+h(t, x(\xi(t)))+f\left(t, x(\xi(t)), \varphi\left(\int_{0}^{\beta(t)} g(t, s, x(\eta(s))) d s\right)\right) .
$$

Moreover, the space $B C\left(\mathbb{R}_{+}\right)$is equipped the following norm:

$$
\|(x)\|_{B C\left(\mathbb{R}_{+}\right)}=\|x\|_{\infty} .
$$

We can easily show that the solution of equation (2.44) in $B C\left(\mathbb{R}_{+}\right)$is equivalent to the fixed point of $T$. Obviously $T x$ is continuous function for any $x \in B C\left(\mathbb{R}_{+}\right)$. Furthermore, using the triangular inequality and 
$\zeta(x(t), 0) \geq 0$ for $t \in \mathbb{R}_{+}$, and additionally by means of our assumptions we obtain

$$
\begin{aligned}
|T(x)(t)| \leq & |A(t)|+|h(t, x(\xi(t)))-h(t, 0)|+|h(t, 0)|+|f(t, 0,0)| \\
& +\left|f\left(t, x(\xi(t)), \varphi\left(\int_{0}^{\beta(t)} g(t, s, x(\eta(s))) d s\right)\right)-f(t, 0,0)\right| \\
\leq & M_{1}+\frac{1}{2} \psi(|x(\xi(t))|)+M_{3}+\frac{1}{2} \psi(|x(\xi(t))|) \\
& +\theta\left(\left|\varphi\left(\int_{0}^{\beta(t)} g(t, s, x(\eta(s))) d s\right)-\varphi(0)\right|\right)+M_{2} \\
\leq & M_{1}+\psi(|x(\xi(t))|)+M_{3}+M_{2}+\theta\left(\delta\left|\left(\int_{0}^{\beta(t)} g(t, s, x(\eta(s))) d s\right)\right|^{\alpha}\right) .
\end{aligned}
$$

So, from above equation and making use of equations (2.41) and (2.42), we have

$$
\|T x\|_{\infty} \leq M_{1}+M_{2}+M_{3}+\psi\left(\|x\|_{\infty}\right)+\theta\left(\delta M_{4}\right) \leq r_{0} .
$$

Thus, $T$ is well-defined and we obtain $T\left(\bar{B}_{r_{0}}\right) \subset \bar{B}_{r_{0}}$. Further, we prove that the mapping $T: \bar{B}_{r_{0}} \rightarrow \bar{B}_{r_{0}}$ is continuous. Let $x, u \in \bar{B}_{r_{0}}$ such that $\zeta(x(t), u(t)) \geq 0$ for $t \in \mathbb{R}_{+}$and for $\epsilon>0,\|x-u\|_{\bar{B}_{r_{0}}}<\frac{\epsilon}{2}$, then we have

$$
\begin{aligned}
|T(x)(t)-T(u)(t)|= & \mid h(t, x(\xi(t)))+f\left(t, x(\xi(t)), \varphi\left(\int_{0}^{\beta(t)} g(t, s, x(\eta(s))) d s\right)\right) \\
& -h(t, u(\xi(t)))-f\left(t, u(\xi(t)), \varphi\left(\int_{0}^{\beta(t)} g(t, s, u(\eta(s))) d s\right)\right) \mid \\
\leq & \frac{1}{2} \psi(|x(\xi(t))-u(\xi(t))|)+\frac{1}{2} \psi(|x(\xi(t))-u(\xi(t))|) \\
& +\theta\left(\left|\varphi\left(\int_{0}^{\beta(t)} g(t, s, x(\eta(s))) d s\right)-\varphi\left(\int_{0}^{\beta(t)} g(t, s, u(\eta(s))) d s\right)\right|\right) \\
\leq & \psi(\|x-u\|)+\theta\left(\delta\left|\int_{0}^{\beta(t)}(g(t, s, x(\eta(s)))-g(t, s, u(\eta(s)))) d s\right|^{\alpha}\right) .
\end{aligned}
$$

Now using the equation (2.43) there exists $T>0$ such that if $t>T$, Then we have

$$
\theta\left(\delta\left|\int_{0}^{\beta(t)}(g(t, s, x(\eta(s)))-g(t, s, u(\eta(s)))) d s\right|^{\alpha}\right) \leq \frac{\epsilon}{2},
$$

for any $x, u \in B C\left(\mathbb{R}_{+}\right)$. Now we consider the following two cases:

Case 1. If $t>T$, then from equations (2.49) and (2.50) we get

$$
|T(x)(t)-T(u)(t)| \leq \psi\left(\frac{\epsilon}{2}\right)+\frac{\epsilon}{2}<\frac{\epsilon}{2}+\frac{\epsilon}{2}=\epsilon .
$$

Case 2. Similarly for $t \in[0, T]$, we have

$$
\begin{aligned}
|T(x)(t)-T(u)(t)| & \leq \psi\left(\frac{\epsilon}{2}\right)+\theta\left(\delta\left|\int_{0}^{\beta(t)}(g(t, s, x(\eta(s)))-g(t, s, u(\eta(s)))) d s\right|^{\alpha}\right) \\
& <\frac{\epsilon}{2}+\theta\left(\delta\left(\beta_{T} \beta(\epsilon)\right)^{\alpha}\right),
\end{aligned}
$$


where $\beta_{T}=\sup \{\beta(t): t \in[0, T]\}$, and

$$
\beta(\epsilon)=\sup \left\{|g(t, s, x)-g(t, s, u)|: t \in[0, T], s \in\left[0, \beta_{T}\right], x, u \in\left[-r_{0}, r_{0}\right],\|x-u\|_{B C\left(\mathbb{R}_{+}\right)}<\frac{\epsilon}{2}\right\} .
$$

Since $g$ is continuous on $[0, T] \times\left[0, \beta_{T}\right] \times\left[-r_{0}, r_{0}\right]$, we have $\beta(\epsilon) \rightarrow 0$ as $\epsilon \rightarrow 0$ and by continuity of $\theta$ we obtain

$$
\theta\left(\delta\left(\beta_{T} \beta(\epsilon)\right)^{\alpha}\right) \rightarrow 0
$$

Finally, from equations (2.51) and (2.52), we conclude that $T$ is a continuous function from $\bar{B}_{r_{0}}$ into $\bar{B}_{r_{0}}$. Now we show that the map $T$ satisfies all the conditions of Theorem 2.11. To do this, for an arbitrary $T>0$ and $\epsilon>0$, assume that $X_{1}$ are arbitrary nonempty subsets of $\bar{B}_{r_{0}}$ and $t_{1}, t_{2} \in[0, T]$, with $\left|t_{1}-t_{2}\right| \leq \epsilon$. Without loss of generality let $\beta\left(t_{1}\right) \leq \beta\left(t_{2}\right)$, and $\zeta\left(x\left(t_{1}\right), x\left(t_{2}\right)\right) \geq 0$, for any arbitrary $x \in X_{1}$, we have

$$
\begin{aligned}
& \left|G x\left(t_{1}\right)-G x\left(t_{2}\right)\right| \\
& =\left|A\left(t_{1}\right)-A\left(t_{2}\right)\right|+\left|h\left(t_{2}, x\left(\xi\left(t_{2}\right)\right)\right)-h\left(t_{2}, x\left(\xi\left(t_{1}\right)\right)\right)\right|+\left|h\left(t_{2}, x\left(\xi\left(t_{1}\right)\right)\right)-h\left(t_{1}, x\left(\xi\left(t_{1}\right)\right) b\right)\right| \\
& +\left|f\left(t_{2}, x\left(\xi\left(t_{2}\right)\right), \varphi\left(\int_{0}^{\beta\left(t_{2}\right)} g\left(t_{2}, s, x(\eta(s))\right) d s\right)\right)-f\left(t_{2}, x\left(\xi\left(t_{1}\right)\right), \varphi\left(\int_{0}^{\beta\left(t_{2}\right)} g\left(t_{2}, s, x(\eta(s))\right) d s\right)\right)\right| \\
& \quad+\left|f\left(t_{2}, x\left(\xi\left(t_{1}\right)\right), \varphi\left(\int_{0}^{\beta\left(t_{2}\right)} g\left(t_{2}, s, x(\eta(s))\right) d s\right)\right)-f\left(t_{1}, x\left(\xi\left(t_{1}\right)\right), \varphi\left(\int_{0}^{\beta\left(t_{2}\right)} g\left(t_{2}, s, x(\eta(s))\right) d s\right)\right)\right| \\
& +\left|f\left(t_{1}, x\left(\xi\left(t_{1}\right)\right), \varphi\left(\int_{0}^{\beta\left(t_{2}\right)} g\left(t_{2}, s, x(\eta(s))\right) d s\right)\right)-f\left(t_{1}, x\left(\xi\left(t_{1}\right)\right), \varphi\left(\int_{0}^{\beta\left(t_{2}\right)} g\left(t_{1}, s, x(\eta(s))\right) d s\right)\right)\right| \\
& \quad+\left|f\left(t_{1}, x\left(\xi\left(t_{1}\right)\right), \varphi\left(\int_{0}^{\beta\left(t_{2}\right)} g\left(t_{1}, s, x(\eta(s))\right) d s\right)\right)-f\left(t_{1}, x\left(\xi\left(t_{1}\right)\right), \varphi\left(\int_{0}^{1} g\left(t_{1}, s, x(\eta(s))\right) d s\right)\right)\right| .
\end{aligned}
$$

Now we make the following substitutions

$$
\left\{\begin{array}{l}
\omega^{T}(A, \epsilon)=\sup \left\{\left|A\left(t_{1}\right)-A\left(t_{2}\right)\right|: t_{1}, t_{1} \in[0, T],\left|t_{1}-t_{2}\right| \leq \epsilon\right\}, \\
\omega_{r_{0}}^{T}(h, \epsilon)=\sup \left\{\left|h\left(t_{2}, x\right)-h\left(t_{1}, x\right)\right|: t_{1}, t_{2} \in[0, T],\left|t_{1}-t_{2}\right| \leq \epsilon, x \in\left[-r_{0}, r_{0}\right]\right\}, \\
\omega^{T}(\xi, \epsilon)=\sup \left\{\left|\xi\left(t_{1}\right)-\xi\left(t_{2}\right)\right|: t_{1}, t_{2} \in[0, T],\left|t_{1}-t_{2}\right| \leq \epsilon\right\}, \\
\omega^{T}\left(x, \omega^{T}(\xi, \epsilon)\right)=\sup \left\{\left|x\left(t_{1}\right)-x\left(t_{2}\right)\right|: t_{1}, t_{2} \in[0, T],\left|t_{1}-t_{2}\right| \leq \omega^{T}(\xi, \epsilon)\right\}, \\
D_{1}=\beta_{T} \sup \left\{|g(t, s, x)|: t \in[0, T], s \in\left[0, \beta_{T}\right], x \in\left[-r_{0}, r_{0}\right]\right\}, \\
\omega_{r_{0}, K}^{T}(f, \epsilon)=\sup \left\{\left|f\left(t_{2}, x, d\right)-f\left(t_{1}, x, d\right)\right|: t_{1}, t_{2} \in[0, T],\left|t_{1}-t_{2}\right| \leq \epsilon,\right. \\
\left.\quad x \in\left[-r_{0}, r_{0}\right], d \in\left[-D_{1}, D_{1}\right]\right\}, \\
\omega_{r_{0}}^{T}(g, \epsilon)=\sup \left\{\left|g\left(t_{1}, s, x\right)-g\left(t_{2}, s, x\right)\right|: t_{1}, t_{2} \in[0, T],\left|t_{1}-t_{2}\right| \leq \epsilon,\right. \\
\left.\quad x \in\left[-r_{0}, r_{0}\right], s \in\left[0, \beta_{T}\right]\right\}, \\
\omega^{T}(q, \epsilon)=\sup \left\{\left|q\left(t_{1}\right)-q\left(t_{2}\right)\right|: t_{1}, t_{2} \in[0, T],\left|t_{1}-t_{2}\right| \leq \epsilon\right\}, \\
U_{r_{0}}^{T}=\sup \left\{|g(t, s, x)|: t \in[0, T], s \in\left[0, \beta_{T}\right], x \in\left[-r_{0}, r_{0}\right]\right\} .
\end{array}\right.
$$


Now using equations (2.54) and (2.55) we have

$$
\begin{aligned}
& \left|T x\left(t_{1}\right)-T x\left(t_{2}\right)\right| \\
\leq & \omega^{T}(A, \epsilon)+\frac{1}{2} \psi\left(\left|x\left(\xi\left(t_{2}\right)\right)-x\left(\xi\left(t_{1}\right)\right)\right|\right)+\omega_{r_{0}}^{T}(h, \epsilon)+\frac{1}{2} \psi\left(\left|x\left(\xi\left(t_{2}\right)\right)-x\left(\xi\left(t_{1}\right)\right)\right|\right)+\omega_{r_{0}, K}^{T}(f, \epsilon) \\
& +\theta\left(\left|\varphi\left(\int_{0}^{\beta\left(t_{2}\right)} g\left(t_{2}, s, x(\eta(s))\right) d s\right)-\varphi\left(\int_{0}^{\beta\left(t_{2}\right)} g\left(t_{1}, s, x(\eta(s))\right) d s\right)\right|\right) \\
& +\theta\left(\left|\varphi\left(\int_{0}^{\beta\left(t_{2}\right)} g\left(t_{1}, s, x(\eta(s))\right) d s\right)-\varphi\left(\int_{0}^{\beta\left(t_{1}\right)} g\left(t_{1}, s, x(\eta(s))\right) d s\right)\right|\right) \\
\leq & \omega^{T}(A, \epsilon)+\omega_{r_{0}}^{T}(h, \epsilon)+\psi\left(\omega^{T}\left(x, \omega^{T}(\xi, \epsilon)\right)\right)+\omega_{r_{0}, K}^{T}(f, \epsilon) \\
& +\theta\left(\delta\left|\int_{0}^{\beta\left(t_{2}\right)}\left(g\left(t_{2}, s, x(\eta(s))\right)-g\left(t_{1}, s, x(\eta(s))\right)\right) d s\right|^{\alpha}\right)+\theta\left(\delta \mid \int_{\beta_{\left(t_{1}\right)}}^{\beta\left(t_{2}\right)}\left(\left.g\left(t_{1}, s, x(\eta(s))\right)\right|^{\alpha}\right)\right. \\
\leq & \left.\omega^{T}(A, \epsilon)+\omega_{r_{0}}^{T}(h, \epsilon)+\psi\left(\omega^{T}\left(x, \omega^{T}(\xi, \epsilon)\right)\right)+\omega_{r_{0}, K}^{T}(f, \epsilon)+\theta\left(\delta\left(\beta_{T} \omega_{r_{0}}^{T}(g, \epsilon)\right)^{\alpha}\right)+\theta\left(\delta K \omega^{T}(\beta, \epsilon)\right)^{\alpha}\right) .
\end{aligned}
$$

Since $x$ is an arbitrary element of $X_{1}$ the above expression implies that

$$
\begin{aligned}
\omega^{L}\left(T\left(X_{1}\right), \epsilon\right) \leq & \omega^{T}(A, \epsilon)+\omega_{r_{0}}^{T}(h, \epsilon)+\psi\left(\omega^{T}\left(X_{1}, \omega^{T}(\xi, \epsilon)\right)\right)+\omega_{r_{0}, K}^{T}(f, \epsilon) \\
& +\theta\left(\delta\left(\beta_{T} \omega_{r_{0}}^{T}(g, \epsilon)\right)^{\alpha}\right)+\theta\left(\delta\left(K \omega^{T}(\beta, \epsilon)\right)^{\alpha}\right) .
\end{aligned}
$$

Moreover, by the uniform continuity of $f, g$ and $h$ on the compact sets $[0, T] \times\left[-r_{0}, r_{0}\right] \times\left[-D_{1}, D_{1}\right],[0, T] \times$ $\left[0, \beta_{T}\right] \times\left[-r_{0}, r_{0}\right]$ and $[0, T] \times\left[-r_{0}, r_{0}\right]$ respectively. We get $\omega_{r_{0}, K}^{T}(f, \epsilon) \rightarrow 0, \omega_{r_{0}}^{T}(g, \epsilon) \rightarrow 0$ and $\omega_{r_{0}, K}^{T}(h, \epsilon) \rightarrow 0$ as $\epsilon \rightarrow 0$. Also due to the uniform continuity of $\xi, q$ and $A$ on $[0, T]$, we get $\omega^{T}(\xi, \epsilon) \rightarrow 0, \omega^{T}(\beta, \epsilon) \rightarrow 0$ and $\omega^{T}(A, \epsilon) \rightarrow 0$ as $\epsilon \rightarrow 0$. Moreover, $\theta$ is a nondecreasing continuous function with $\theta(0)=0$ and $K$ is finite, hence we have $\theta\left(\delta\left(\beta_{T} \omega_{r_{0}}^{T}(g, \epsilon)\right)^{\alpha}\right)+\theta\left(\delta\left(K \omega^{T}(\beta, \epsilon)\right)^{\alpha}\right) \longrightarrow 0$, as $\epsilon \rightarrow 0$. Now taking the limit $\epsilon \rightarrow 0$ in equation (2.57), we get

$$
\omega_{0}^{L}\left(T\left(X_{1}\right)\right) \leq \psi\left(\omega_{0}^{T}\left(X_{1}\right)\right),
$$

and taking the limit $T \rightarrow \infty$ in aforementioned equation (2.58), we obtain

$$
\omega_{0}\left(T\left(X_{1}\right)\right) \leq \psi\left(\omega_{0}\left(X_{1}\right)\right) .
$$

Further, in addition $\zeta(x(t), u(t)) \geq 0$ for arbitrary $x, u, \in X_{1}$

$$
\begin{aligned}
|T x(t)-T u(t)| \leq & |h(t, x(\xi(t)))-h(t, u(\xi(t)))| \\
& +\left|f\left(t, x(\xi(t)), \varphi\left(\int_{0}^{\beta(t)} g(t, s, x(\eta(s))) d s\right)\right)-f\left(t, u(\xi(t)), \varphi\left(\int_{0}^{\beta(t)} g(t, s, u(\eta(s))) d s\right)\right)\right| \\
\leq & \frac{1}{2} \psi(|x(\xi(t))-u(\xi(t))|)+\frac{1}{2} \psi(|x(\xi(t))-u(\xi(t))|) \\
& +\theta\left(\left|\varphi\left(\int_{0}^{\beta(t)} g(t, s, x(\eta(s))) d s\right)-\varphi\left(\int_{0}^{\beta(t)} g(t, s, u(\eta(s))) d s\right)\right|\right) \\
\leq & \psi\left(\operatorname{diamX}_{1}(\xi(t))\right)+\theta\left(\delta\left|\int_{0}^{\beta(t)}(g(t, s, x(\eta(s)))-g(t, s, u(\eta(s)))) d s\right|^{\alpha}\right) .
\end{aligned}
$$

Since $x, u$ and $t$ are arbitrary in equation (2.60) we conclude that

$$
\operatorname{diam} T\left(X_{1}\right) \leq \psi\left(\operatorname{diam} X_{1}(\xi(t))\right)+\theta\left(\delta\left|\int_{0}^{\beta(t}(g(t, s, x(\eta(s)))-g(t, s, u(\eta(s)))) d s\right|^{\alpha}\right) .
$$


Taking $t \rightarrow 0$ in equation (2.61) and using equation (2.43), we have

$$
\underset{t \rightarrow \infty}{\limsup \operatorname{diam}} \operatorname{TX}_{1}(t) \leq \psi\left(\limsup _{t \rightarrow \infty} \operatorname{diam}_{1}(\xi(t))\right) .
$$

Now from equations (2.59), (2.62) and taking into account the superadditivity of the function $\psi$, we conclude that

$$
\begin{aligned}
\omega_{0}\left(T\left(X_{1}\right)+\limsup _{t \rightarrow \infty} \operatorname{diam} T\left(X_{1}\right)(t)\right. & \leq \psi\left(\omega_{0}\left(X_{1}\right)\right)+\psi\left(\limsup _{t \rightarrow \infty} \operatorname{diam} X_{1}(\xi(t))\right) \\
& \leq \psi\left(\omega_{0}\left(X_{1}\right)+\limsup _{t \rightarrow \infty} \operatorname{diam} X_{1}(\xi(t))\right)
\end{aligned}
$$

Finally, from equation (2.34), we get

$$
\mu\left(T\left(X_{1}\right)\right) \leq \psi\left(\mu\left(X_{1}\right)\right) .
$$

Furthermore, we define the function $\beta: B C\left(\mathbb{R}_{+}\right) \times B C\left(\mathbb{R}_{+}\right) \longrightarrow[0,+\infty)$, by

$$
\beta\left(X_{1}\right)= \begin{cases}1 & \zeta(x(t), y(t)) \geq 0, \forall x, y \in X_{1}, \\ 0 & \text { otherwise, }\end{cases}
$$

which implies that for any $X_{1} \subseteq B_{r_{0}}$, we have

$$
\beta\left(X_{1}\right) \mu\left(T\left(X_{1}\right)\right) \leq \psi\left(\mu\left(X_{1}\right)\right) .
$$

Let $\beta(X) \geq 1$, which implies that $\zeta(x(t), y(t)) \geq 0$, and from our assumption (viii) implies that $\beta(\overline{c o} T X) \geq 1$, so $T$ is $\beta$-admissible. Since from hypothesis $\zeta\left(x_{0}(t), y_{0}(t)\right) \geq 0$, for all $x_{0}, y_{0} \in B_{r_{0}}$, implies that $\beta\left(B_{r_{0}}\right) \geq 1$. Thus by Theorem 2.11, $T$ has atleast one fixed point in $B C\left(\mathbb{R}_{+}\right)$.

\section{Conclusion}

Taking into account its interesting applications, looking for newly fixed point theorems concerning the new setup of contractive type conditions has acquired considerable attention over the last few decades. In this regard, the main purpose of this paper is to provide new ideas of $\alpha-\psi$ and $\beta-\psi$ condensing operators and make use of them to establish a new fixed point and coupled fixed point theorems. An application to a solution of the functional integral equation is illustrated to the usability of the obtained fixed point results.

Acknowledgements: This project was supported by Theoretical and Computational Science (TaCS) Center under Computational and Applied Science for Smart Innovation Cluster (CLASSIC), Faculty of Science, KMUTT. Habib ur Rehman was supported by the Petchra Pra Jom Klao Doctoral Scholarship Academic for PhD Program at KMUTT. Moreover, this research work was financially supported by King Mongkut's University of Technology Thonburi through the "KMUTT $55^{\text {th }}$ Anniversary Commemorative Fund".

\section{References}

[1] Brouwer L. E. J., Über abbildung von mannigfaltigkeiten, Math. Ann., 1911, 71(1), 97-115

[2] Banach S., Sur les opérations dans les ensembles abstraits et leur application aux équations intégrales, Fund. Math., 1922, 3(1), 133-181

[3] Schauder J., Der Fixpunktsatz in Funktional-raumen, Studia Math., 1930, 2(1), 171-180

[4] Banaś J., On measures of noncompactness in Banach spaces, Comment. Math. Univ. Carolin., 1980, 21(1), 131-143

[5] Banaś J., Applications of measures of weak noncompactness and some classes of operators in the theory of functional equations in the Lebesgue space, Nonlinear Anal., 1997, 30(6), 3283-3293

[6] Belluce L., Kirk W., Fixed-point theorems for families of contraction mappings, Pacific J. Math., 1966, 18(2), 213-217

[7] Belluce L. P., Kirk W. A., Nonexpansive mappings and fixed-points in Banach spaces, Illinois J. Math., 1967, 11(3), 474-479

[8] Edelstein M., An extension of Banach's contraction principle, Proc. Amer. Math. Soc., 1961, 12(1), 7-10 
[9] Samet B., Vetro C., Vetro P., Fixed point theorems for $\alpha-\psi$-contractive type mappings, Nonlinear Anal., 2012, 75(4), 21542165

[10] Bhaskar T. G., Lakshmikantham V., Fixed point theorems in partially ordered metric spaces and applications, Nonlinear Anal., 2006, 65(7), 1379-1393

[11] Kuratowski C., Sur les espaces complets, Fund. Math., 1930, 1(15), 301-309

[12] Heinz H. P., On the behaviour of measures of noncompactness with respect to differentiation and integration of vector-valued functions, Nonlinear Anal., 1983, 7(12), 1351-1371

[13] Appell J., Measures of noncompactness, condensing operators and fixed points, an application-oriented survey, Fixed Point Theory, 2005, 6(2), 157-229

[14] Aghajani A., Banaś J., Sabzali N., Some generalizations of Darbo fixed point theorem and applications, Bull. Belg. Math. Soc. Simon Stevin, 2013, 20(2), 345-358

[15] Aghajani A., Haghighi A. S., Existence of solutions for a system of integral equations via measure of noncompactness, Novi Sad J. Math., 2014, 44(1), 59-73

[16] Aghajani A., Mursaleen M., Haghighi A. S., Fixed point theorems for Meir-Keeler condensing operators via measure of noncompactness, Acta Math. Sci., 2015, 35(3), 552-566

[17] Arab R., Some generalizations of Darbo fixed point theorem and its application, Miskolc Math. Notes, 2017, 18(2), 595-610

[18] Cai L., Liang J., New generalizations of Darbo's fixed point theorem, Fixed Point Theory Appl., 2015, 156

[19] Aghajani A., Jalilian Y., Existence and global attractivity of solutions of a nonlinear functional integral equation, Commun. Nonlinear Sci. Numer. Simul., 2010, 15(11), 3306-3312

[20] Aghajani A., Jalilian Y., Existence of nondecreasing positive solutions for a system of singular integral equations, Mediterr. J. Math., 2011, 8(4), 563-576

[21] Banaś J., Rzepka B., An application of a measure of noncompactness in the study of asymptotic stability, Appl. Math. Lett., 2003, 16(1), 1-6

[22] Darwish M. A., Henderson J., O’Regan D., Existence and asymptotic stability of solutions of a perturbed fractional functionalintegral equation with linear modification of the argument, Bull. Korean Math. Soc., 2011, 48(3), 539-553

[23] Dhage B., Bellale S. S., Local asymptotic stability for nonlinear quadratic functional integral equations, Electron. J. Qual. Theory Differ. Equ., 2008, 10, 1-13

[24] Mursaleen M., Arab R., On existence of solution of a class of quadratic-integral equations using contraction defined by simulation functions and measure of noncompactness, Carpathian J. Math., 2018, 34(3), 371-378

[25] Banaś J., Mursaleen M., Sequence spaces and measures of noncompactness with applications to differential and integral equations, 1st ed, Springer, New Delhi, 2014

[26] Das A., Hazarika B., Arab R., Mursaleen M., Solvability of the infinite system of integral equations in two variables in the sequence spaces $c_{0}$ and $l_{1}$, J. Comput. Appl. Math., 2017, 326, 183-192

[27] Zhang J., Sun J., Exponential synchronization of complex networks with continuous dynamics and Boolean mechanism, Neurocomputing, 2018, 307, 146-152

[28] Kreyszig E., Introductory functional analysis with applications, Wiley, New York, 1978

[29] Darbo G., Punti uniti in trasformazioni a codominio non compatto, Rend. Sem. Mat. Univ. Padova., 1955, 24, 84-92

[30] Akhmerov R. R., Kamenskii M. I., Patapov A. S., Rodkina A. E., Sadovskii B. N., Measures of noncompactness and condensing operators, 1st ed, Birkhäuser Basel, Springer Basel AG, 1992

[31] Appell J., Banaś J., Merentes N., Measures of noncompactness in the study of asymptotically stable and ultimately nondecreasing solutions of integral equations, Z. Anal. Anwend., 2010, 29(3), 251-273

[32] Toledano J. M., Benavides T. D., Acedo G. L., Measures of noncompactness in metric fixed point theory, 1st ed, Birkhäuser Basel, Springer Basel AG, 1997 